\title{
Fibre-optic probe hydrophone for ultrasonic and shock-wave measurements in water
}

\author{
J. Staudenraus and W. Eisenmenger \\ Universität Stuttgart, 1. Physikalisches Institut, Pfaffenwaldring 57, D-7000 Stuttgart 80 , \\ Germany
}

Received 16 October 1992; accepted 28 December 1992

\begin{abstract}
Aimed at lithotripter acoustic output measurements, a new fibre-optic probe hydrophone overcomes most of the problems involved with the use of piezoelectric hydrophone technology in non-linear ultrasonic and shock-wave fields. The fibre-optic principle allows for extremely wide bandwidth (larger than $1 \mathrm{GHz}$ ) and superior electromagnetic shielding. Contrary to hitherto existing hydrophones a high cavitation threshold at the water-silica interface provides undistorted detection of strong rarefractional pulse pressures. Considering pure compression there is good agreement between maximum pulse pressure derived from fibre-optic hydrophone theory and the corresponding amplitudes obtained from acoustically calibrated PVDF membrane and needle hydrophones.
\end{abstract} Keywords: hydrophones; fibre optics; piezoelectrics; lithotripsy; shock waves;
cavitation

Miniature needle and membrane hydrophones having polyvinylidene fluoride (PVDF) sensitive elements are widely accepted for measurements in ultrasonic fields ${ }^{1-6}$. Assuming plane waves, commercially available thin polymer films $(9 \mu \mathrm{m})$ are used to construct broadband detectors up to $100 \mathrm{MH}$. Unfortunately, 'fringe' effects associated with spot poling PVDF films make sensor element diameters below $0.5 \mathrm{~mm}$ difficult to realize? Therefore, in focussing fields, due to coupling of the space and time resolution for curved waves, a severe bandwidth limitation must be considered.

The demand for precise measurements in high-intensity focussing fields has increased within the past decade as extracorporeal shock-wave lithotripsy for non-surgical treatment of renal and biliary stones has become established. High pressure amplitudes in the $100 \mathrm{MPa}$ range, exceeding the amplitudes of diagnostic ultrasound by a factor 10 to 100 , imply strong non-linear wave propagation. Available data from plane $10 \mathrm{MPa}$ pressure pulses in water confirm that non-lincar steepening stabilizes shock fronts with rise times in the order of $1 \mathrm{~ns}$ (see Reference 8).

Under free field focussing conditions, as are realized by lithotripter sources, even a pure compressional pulse is followed by a diffraction induced rarefractional phase. On-axis negative pressure amplitudes exceed $-10 \mathrm{MPa}$, and are accompanied by intense cavitation ${ }^{9}$.

In the meantime, several authors have presented lithotripter field measurements using PVDF needle and membrane hydrophones ${ }^{10-12}$. Improvement against cavitation damage could be achieved by cavitation guard materials ${ }^{13,14}$ as well as by separating the electrodes from the active layer, forming capacitively coupled devices ${ }^{15}$. However, signal distortions related to the acoustic field pertubation introduced by the pressure sensors have not been taken into account. In addition, according to recently published results ${ }^{14.16}$, a low cavitation threshold on metallized PVDF surfaces limits the detectable negative pressure.

In 1988 we presented a new fibre-optic probe hydrophone ${ }^{17}$ which was further developed to become a practicable measurement tool. We describe now a compact transportable device, giving a signal bandwidth of $20 \mathrm{MHz}$ and a spatial resolution of $0.1 \mathrm{~mm}$, which overcomes most of the problems involved with the use of piezoelectric measurcment technology in high intensity sound fields.

As an acoustic sensing element, the endface of a glass or polymer fibre is introduced into the acoustic wavefield. A temporal pressure change gives a temporal variation of the optical reflectance at the boundary between the fibre endface and liquid, and this is used as a hydrophone signal. The optical reflectance at the fibre endface is linked with the pressure amplitude via the index of refractiondensity relationship. When the pressure increases, the density, and hence the refractive indices of the liquid and the fibre, are increased. However, due to the low compressibility of the solid fibre material the change of the index of refraction in the liquid prevails. The resulting change of the optical reflectance is registered photoelectrically via the time-dependent intensity of the reflected light at a constant irradiation level.

\section{Theory}

The coefficient of refiection as a function of pressure is derived from the Fresnel formulas, the index of refraction-density relations and the equation of state of the media.

In commercial step index silica fibres having core 


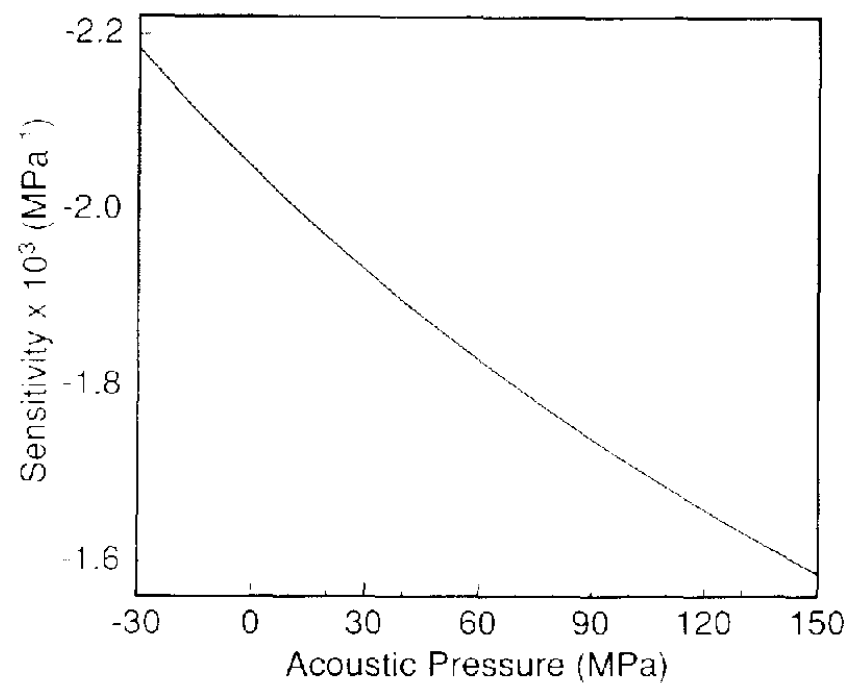

Figure 1 Sensitivity of a fibre-optic probe hydrophone in water $\left(20 \mathrm{C}_{c} ; \cdots-800 \mathrm{~nm}\right.$, silica fibre $n_{\mathrm{c}}=1.483$ ). evaluated from (1) (4). Further parameters according to text

diameters between $100 \mu \mathrm{m}$ and $200 \mu \mathrm{m}$ light propagation is distributed to typically $10^{3}$ to $10^{4}$ light guiding modes, forming angles $<15$ to the fibre axis. With a uniform distribution on the modes, as well as on both polarization orientations considered, the coefficient of reflection, $R$, at a flat perpendicular endface is well approximated by the reflection coefficient of the perpendicular mode:

$R=\left[\left(n_{c}-n_{\mathrm{w}}\right):\left(n_{\mathrm{c}}+n_{\mathrm{u}}\right)\right]^{2}$

$n_{c}=$ index of refraction of the fibre-core

$n_{w}=$ index of refraction of the fluid (water)

The index of refraction and pressure are connected by the density $\rho$. The change of state in a shock front is given by the Hugoniot curve and may be approximated in the MPa-range by Poisson's law ${ }^{i x}$. In water up to $1 G P_{a}$, the isentropic Tait equation

$(P+Q): p^{\prime}=$ constant :

$$
P=P_{1}+p
$$$$
P_{0}=\text { static pressure }: p=\text { acoustic pressure }
$$

holds. using fit parameters $Q=295.5 \mathrm{MPa}$ and $\eta^{\prime}=7.44$. evaluated for standard conditions $\left(T=20 \mathrm{C}, P_{0}-\right.$ $\left.100 \mathrm{kPa} \rho=1000 \mathrm{~kg} \mathrm{~m}^{3}\right)^{19.20}$.

The relation between density and index of refraction for water is well fit by the Gladstone Dale model at dynamic compressions up to approximately $500 \mathrm{MPa}$ (see References 21,22 ), confirming the results obtained from static compression ${ }^{2.3}$ within an error limit of $5 \%$ :

$\left(n_{\mathrm{w}}-1\right) ; \rho-$ constant

Evaluating the pressure dependent change in the index of refraction from Equations (2) and (3) results, for water and a light wavelength $\lambda=800 \mathrm{~nm}\left(n_{w}=1.329\right)$, in $\Delta n_{\mathrm{w}}: \Delta p \approx 1.4 \times 10^{-4} \mathrm{MPa}^{-1}$, which is in accordance with experimental data ${ }^{22}$. The corresponding value for a silica fibre is given by $\Delta n_{\mathrm{c}} \Delta p \approx 5 \times 10^{-6} \mathrm{MPa}^{-1}$ (Reference 24). Hence, for simplicity we consider $n_{\mathrm{c}}$ to be constant with regard to the compressibility of the fibre by a final pressure data correction of $+3.6 \%$ an approximation which holds for $n_{\mathrm{c}} \approx n_{\mathrm{w}}$.

The sensitivity $H$ of the hydrophone is now defined by the change in light reflectivity $\Delta R$. divided by the acoustic pressure $p$ and the static reflectivity $R_{0}$. S accounts for additional stray light due to non-ideal experimental conditions:

$\Delta-c$
$\left(R_{01}+S\right) p$

Figure / gives the hydrophone sensitivity plotted from Equations (1) (4), assuming an incompressible fibre and $S=0$ ). For pressure amplitudes between 5 and +30 MPa the non-linear aberration from an average sensitivity of $-1.95 \times 10^{-3} \mathrm{MPa}^{-1}$ is helow $5 \%$. Between $20 \mathrm{C}$ and $40 \mathrm{C}$ the hydrophone sensitivity decreases by $3 \times 10^{-1}$ per degree rise of temperature. Higher sensitivities may be obtained by using media with larger changes of the refraction index with pressure ${ }^{25}$, via a beam path close to the critical angle of total reflection ${ }^{20.27}$, by reducing the difference of the refractive indices between the waveguide and liquid as well as by selecting the light frequency $r$ on the edge of a molecular transition of oscillation or an electronic transition. whereby a pressure shift of the respective optical transition leads to large changes of the refractive index. These possibilities, which simultaneously cause it deterioration of the linear behaviour, are not necessary in the regime of shock waves. In addition, the final sensitivity limit, as determined by the signal-to-noise ratio, can be significantly improved by reduction of the laser noise.

A principal limit is set by the photon noise. For a signal-to-noise ratio of unity (photon shot noise) the minimum detectable pressure $p_{\min }$ results in

$p_{\min }=\left(\begin{array}{c}2\left(R_{0}+S\right) h \Lambda f \\ q W_{0}\end{array}\right)^{1.2} \cdot\left(\begin{array}{l}\mathrm{d} R \\ \mathrm{~d} p\end{array}\right)_{n}$

The low noise opcration of the hydrophone, due to the desired large bandwidth $\Delta f$, requires a light source with high photon flux $W_{0} / h$, with $W_{0}$ of the order of 0.1 to $1 \mathrm{~W}$. as well as a photodetector with high quantum yield $q$ and the amount of scattered light $S$ to be small compared with the static reflection $R_{0}$.

The sensitive volume of the optical probe hydrophone is formed by the cross-section of the fibre core and half of the optical wavelength. This corresponds to a maximum bandwidth of typically $3 \mathrm{GHz}$ with perpendicular acoustic wave incidence in water.

\section{Experimental set-up}

In our first experimental set-up, reported in 1988 ${ }^{\circ}$, we used a water-cooled argon laser and coupled light via a microscope objective into a $200 / 240 \mu \mathrm{m}$ step-index silicat fibre.

In the meantime, a widely improved, small, rugged and easy to handle device has been constructed. Figurc ? illustrates the optical and experimental arrangement. For convenience we chose a $100: 140 \mu \mathrm{m}$ step-index silicat fibre. A $100 \mathrm{~mW}$ fibre-pigtailed continuous-wave diode laser $(\lambda=800 \mathrm{~nm})$ is spliced to port 1 of a $3 \mathrm{~dB}$ fibre coupler. The light coming from port 2 is guided by the libre to the hydrophone probe tip in the measurement position inside the water lank. Using professional cleaving tools, the fibre endface is cut perpendicular to the libre axis. From this perfectly clean and highly reproducible endface a small a mount of the incident light. intensity modulated by the acoustic signal. is reflected 


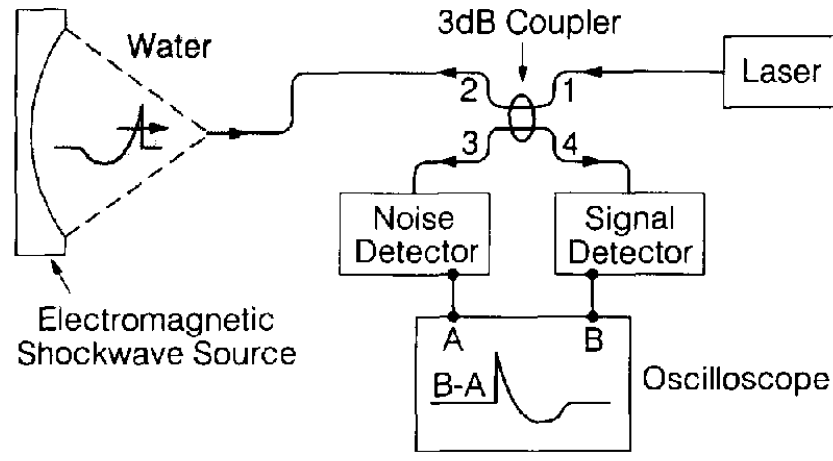

Figure 2 Fibre-optic probe hydrophone: experimental set-up

back and detected by a silicon $\mathrm{p}-\mathrm{i}-\mathrm{n}$ photodiode via port 4 of the coupler. Transimpedance amplification gives a bandwidth from dc to $20 \mathrm{MHz}$. Finally, noise caused by coherent laser amplitude fluctuation is compensated by subtracting the noise signal derived from port 3 . A properly designed noise detector avoids any light reflection, minimizing stray light $S$.

Optical signal transmission along the non-conductive silica fibre, attached to the battery driven laser source and detectors, provides superior electromagnetic shielding. Hence, electrical disturbances caused by the spark discharge involved with the generation of shock waves are mostly suppressed. Denoting $U_{0}$ as the hydrophone voltage signal corresponding to static reflection $R_{0}+S$, the noise level $U_{\text {noise }} / U_{0} \approx 2 \times 10^{-3}$ (single shot, $20 \mathrm{MHz}$ bandwidth) approaches the theoretical shot noise limit by a factor of 2 . Since the minimum detectable pressure is experimentally determined by the quotient of noise level and sensitivity (from Figure 1), this results in $p_{\min } \approx 1 \mathrm{MPa}$, respectively $\pm 0.5 \mathrm{MPa}$ resolution.

\section{Static calibration of the fibre-optic probe hydrophone}

Keeping in mind that the hydrophone sensitivity is rather low compared with piczoelectric polymer hydrophones, yet it does not depend on the fibre core diameter, acoustic calibration techniques can be performed in step or sinus wave fields of known high amplitude. This is possible with electromagnetically generated pressure pulses using the discharge current and the generator coil winding density for amplitude calculation ${ }^{28}$, or with mechanical shock tubes or with indirect pressure determination by other transducers acoustically calibrated at lower wave amplitudes.

The fibre-optic probe hydrophone, by virtue of its inherent, almost unlimited, bandwidth starting from dc, provides the unique feature of a simple static-wideband optical calibration. According to the hydrophone theory, the sensitivity Equation (4) can be computed from the fibre and liquid data under inclusion of the unknown stray light $S$. In detail, the previously denoted hydrophone sensivitiy is written in terms of the corresponding hydrophone voltage signals $\Delta U$ and $U_{0}$ at the output of the $\mathrm{p}-\mathrm{i}-\mathrm{n}$ photodiode :

$\frac{\Delta U}{U_{0} p}=\frac{\Delta R}{\left(R_{0}+S\right) p}$

$\Delta U=$ pressure pulse voltage signal

$U_{0}=\mathrm{dc}$ reflectance voltage signal with the fibre immersed into the liquid
$S$ can be determined under 'zero reflectivity' conditions - for instance, by immersing the fibre endface into a index-adapted fluid. A typical value from our hydrophone is $S \approx 3 \times 10^{-2} R_{0}$, caused by internal crosstalk of the fibre coupler. Therefore, in routine measurement $U_{0}$ gives the complete (static) calibration.

An additional check with respect to $S$ and $R_{0}$ is provided by measuring the signal voltage $U_{0}^{*}$ for the probe light reflection in air. Calculation of $U_{0}^{*}$ under ideal conditions ( $S=0$, dry air ) results in $U_{0}^{*}=x U_{0}$ with $\alpha=12.6$. Lower values of $\alpha$ indicate increased $S$ values by contamination of the fibre tip.

\section{Step wave calibration of hydrophones}

Despite the virtue of static calibration it is essential to investigate the dynamic properties of a hydrophone by means of the complete amplitude and phase or, equivalently, the step or delta-pulse response. Considering shock wave pressure-time functions, the step wave calibration is particularly appropriate for an immediate control of geometrical reflection and refraction properties as well as probe resonances. The true shock-wave form is obtained by deconvolution of the observed signal using the step wave response of the hydrophone.

Experimentally, we create a pressure step by the transicnt excitation of a thick piezoelectric disc. This standard acoustic source radiating a rectangular-shaped plane wave of approximately $50 \mathrm{kPa}$ into water, simultaneously provides an absolute calibration from electrical parameters 29 .

Figures 3-5 show the signal recorded from the first period of the rectangular-shaped plane wave (rise time $50 \mathrm{~ns}$ ) for three different hydrophones. Due to its good acoustic transparency, the membrane hydrophonc, Figure 3, records the radiated wave almost undistorted (24 $\mu \mathrm{m}$ PVDF, spot poled piezoelectric diameter $0.8 \mathrm{~mm}$, vacum evaporated gold electrodes ${ }^{14}$ ). The PVDF needle

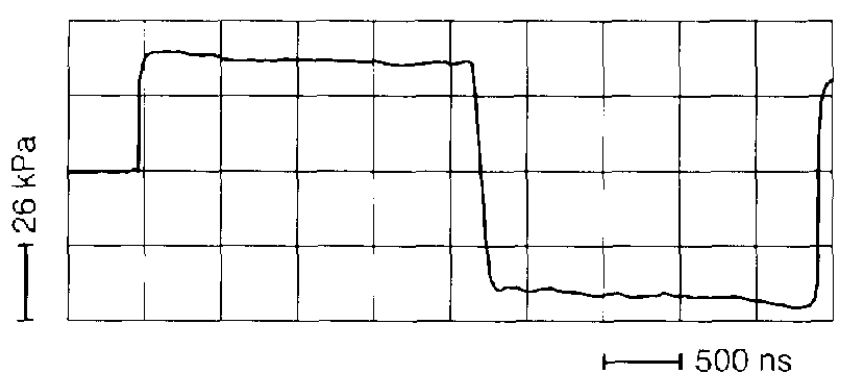

Figure 3 PVDF membrane hydrophone: signal response due to a rectangular shaped plane wave

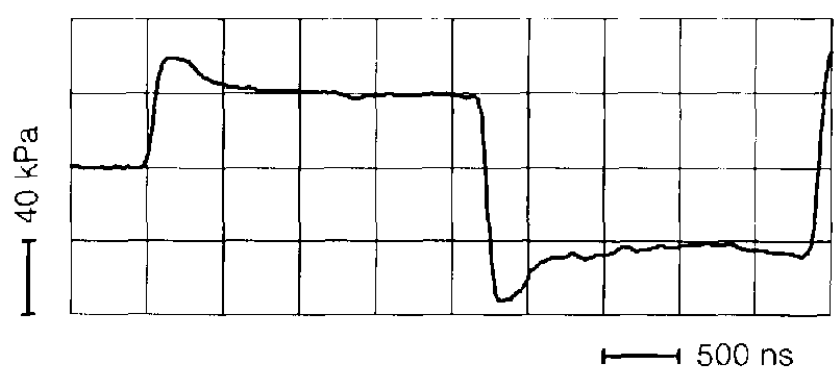

Figure 4 Commerical PVDF needle hydrophone $e^{6}$ : signal corresponding to Figure 3 


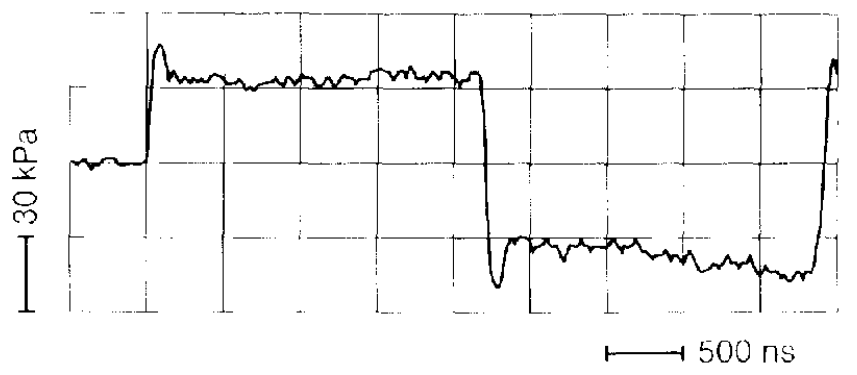

Figure 5 Fibre-optic probe hydrophone (sensitivity enhanced by $100 \mu \mathrm{m}$ optically clear silicone rubber, covering fibre endface) signal corresponding to Figure 3

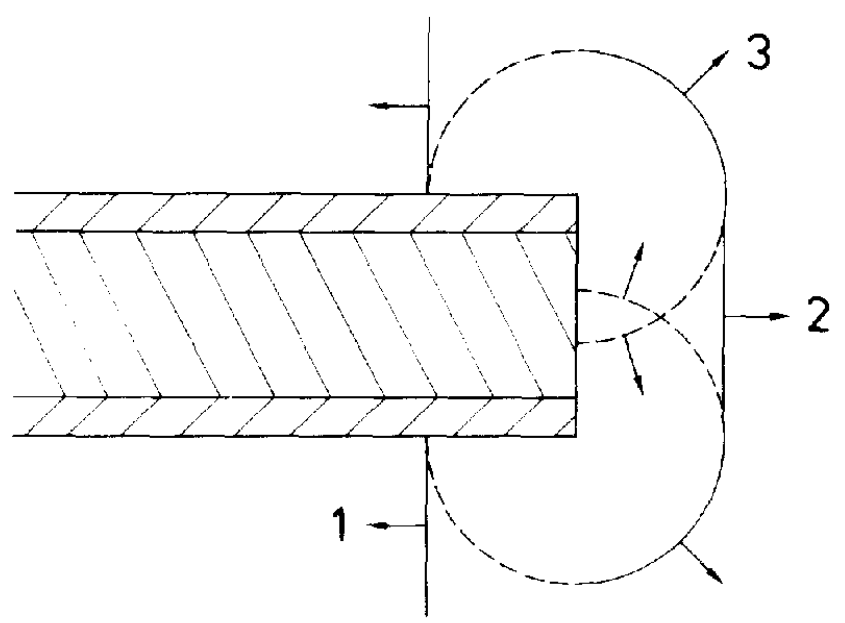

Figure 6 Plane compression step incident to cylindrical probe hydrophone: incident wave (1), acoustically rigid reflected wave (2), diffracted edge wave (3): compression, rarefraction

hydrophone", having a hemispherical tip of $1 \mathrm{~mm}$ in diameter (spot poled piezoclectric zone restricted to about $0.5 \mathrm{~mm})$, indicates acoustically rigid rellection from the lip by a characteristic signal overshoot. Figure 4. decaying within $500 \mathrm{~ns}$. A corresponding signal behaviour is observed for the fibre-optic probe hydrophone, Figure 5 : however, owing to its miniature design $(100 / 140 / \mathrm{m}$ fibre $)$, the sensitivity approaches a constant level within $80 \mathrm{~ns}$. In this case, signal averaging and increasing the hydrophone sensitivity by covering the libre endface with a $100 \mu \mathrm{m}$ layer of optically elear silicone rubber ${ }^{25}$ has been used to improve the signal-to-noise ratio.

A distortion of the initial step generally observed from probe hydrophone recordings ${ }^{14.16}$ is caused essentially by secondary waves emerging from the hydrophone. The resulting pressure field (Figure 6 ) consists of the incident wave, the reflected wave and the wave diffracted at the hydrophone edge. In the case of a pressure step. the pressure being detected for the first moment amounts to the double of the incident wave, due to acoustically rigid reflection at the hydrophone tip. However. alter the phase-inverted edge wave has completely propagated across the sensitive area, the resulting pressure is reduced to the pressure of the incident wave.

\section{Shock front response of different hydrophones}

Figures 7 to 9 present shock lront signals recorded by the different hydrophones characterized above. successively positioned in the focus of our self-focussing electromagnetic shock wave source ${ }^{14.16 .28}$. Looking at the fibre-optic probe hydrophone signal Figure 7 (bare fibre), the shock front is accompanied by a $80 \mathrm{~ns}$ wide overshoot, consistent with the rectangular wave response. From the membrane hydrophone, see Fiqure 8 , we observe high frequency oscillations, due to multiple reflections inside the $24 \mu \mathrm{m}$ PVDF membrane, which are slightly distorted by a low sampling rate $\left(250 \mathrm{Ms} \mathrm{s}^{-1}\right)$. The PVDF needle hydrophone signal Figure" oxhibits a comparatively slow rise time and strong waveform distortion. introduced by the hydrophone geometry.

A reconstruction of the true acoustic waveform can be

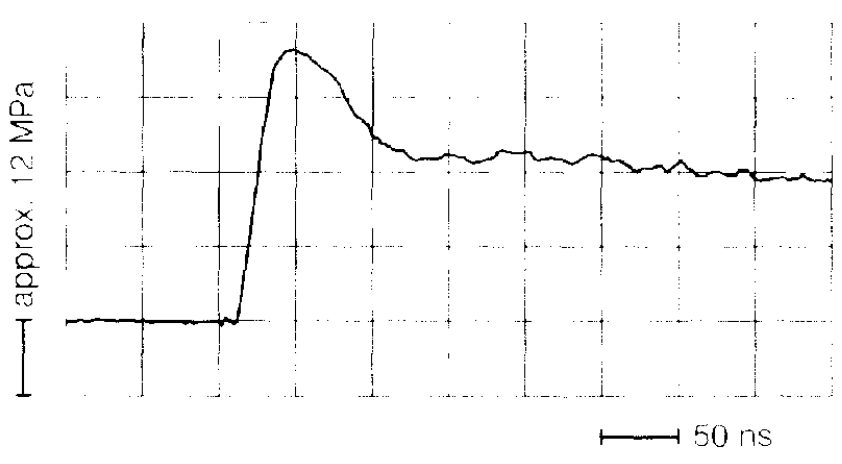

Figure 7 Self-focussing electromagnetic source ${ }^{1:}$ : shock tront signal recorded in the geometrical focus by a fibre-optic probe hydrophone: rise time $17 \mathrm{~ns}$, limited by amplifier bandwidth $20 \mathrm{MHz}$ : linearization and high frequency linear extrapolation sets shock front amplitude to $-30 \mathrm{MPa}+5 \%$

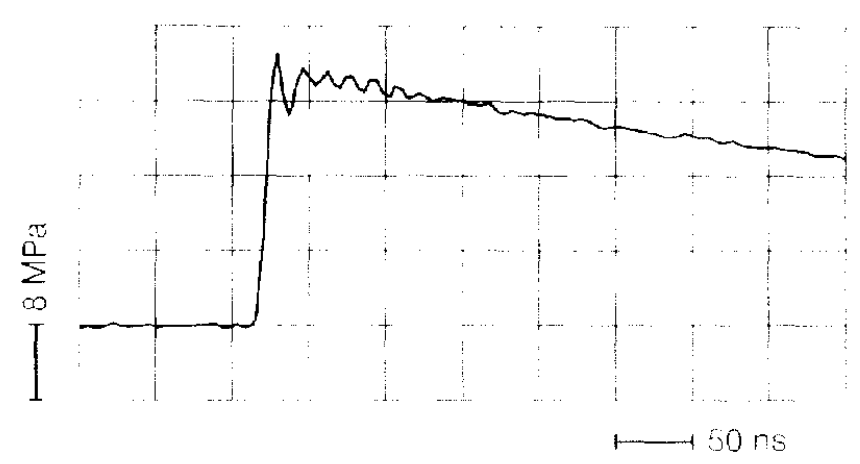

Figure 8 Shock front signal corresponding to Figure 7, but PVDF membrane hydrophone: rise time $8 \mathrm{~ns}$, limited by membrane thickness $(24 \mathrm{um})$; high trequency linear extrapolation sets shock front amplitude to $28 \mathrm{MPa}+10 \%$ (including calibration accuracy $+5 \%)$

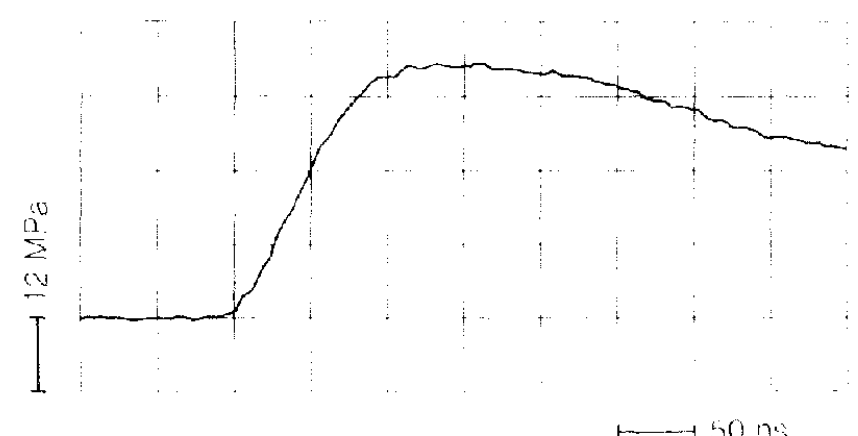

Figure 9 Shock front signal corresponding to tigure $t$, but commercial PVDF necdle hydrophone ${ }^{6}$. rise time $74 \mathrm{~ns}$. limited by hemispherical geometry: deconvolution sets shock front amplitude to $31 \mathrm{MPa}+15 \%$ (including calibration accuracy $+5 \%$ ) 


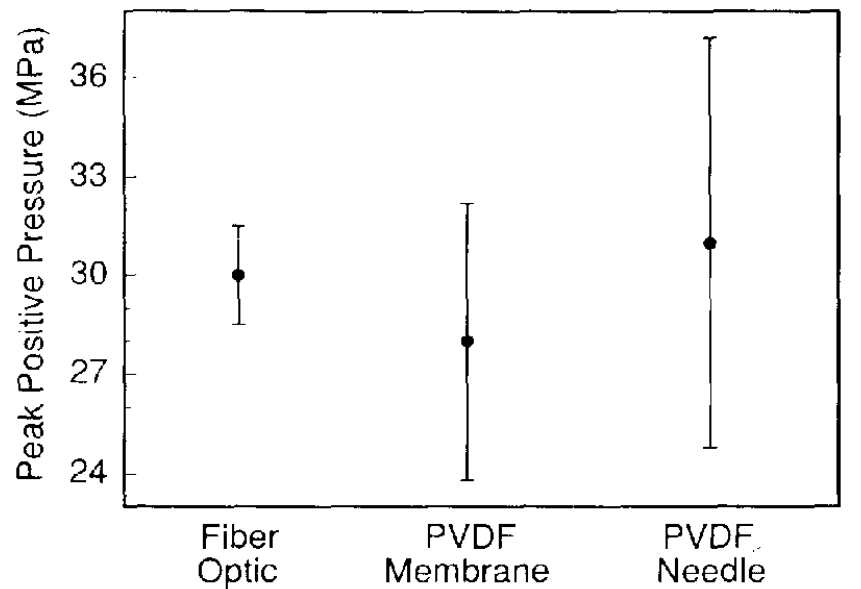

Figure 10 Shock front amplitudes as measured by different hydrophones in the geometrical focus of a self-focussing electromagnetic source, resulting from signal analysis in Figures 7 - 9

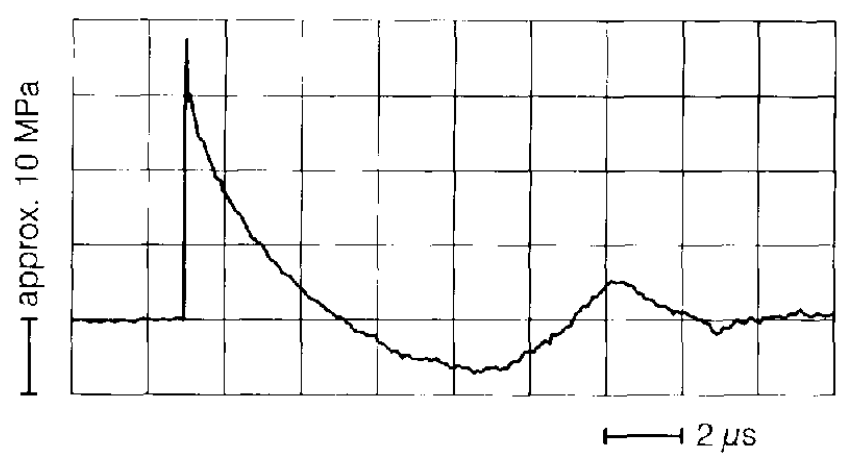

Figure 11 Self-focussing electromagnetic source ${ }^{14}$ : focal signal at maximum setting, recorded by a fibre-optic probe hydrophone: linearization and high frequency linear extrapolation sets extrema to $+30 /-7.2 \mathrm{MPa} \pm 5 \%$

obtained within the signal-to-noise limits .. for all hydrophones - by deconvolution of the linearized signal and the impulse response. In this analysis, the true shock front rise-time in the $1 \mathrm{~ns}$ regime cannot, of course, be resolved since the experimental impulse response function is limited to rise-times of the order of $20 \mathrm{~ns}$. With respect to medical applications, this appears to be of less importance.

Refcrring to the fibre-optic probe hydrophone signal (Figure 7) and to the membrane hydrophone signal (Figure 8), linear extrapolation of the almost linear decaying waveform, which cuts off the $80 \mathrm{~ns}$ wide overshoot and respectively averages the high frequency oscillations, results in a close approximation of the true shock front amplitude. That simple procedure however fails with the PVDF needle hydrophone signal (Figure 9). After the appropriate signal analysis we find a good agreement between maximum shock pressure derived from fibre-optic hydrophone theory and PVDF hydrophone calibration (see Figure 10).

\section{Comparison of the negative pressure response of different hydrophones}

In analysing the complete focal waveform of our highly reproducible electromagnetic shock-wave source, it turns out that the fibre-optic probe hydrophone (Figure 11 ) registers a long negative pressure tail without cavitation, which would show up by a sharp reflectivity increase and a corresponding negative signal jump ${ }^{14,16}$. In contrast, PVDF membrane hydrophones (Figure 12) as well as PVDF needle hydrophones (Figure 13), detect only the leading edge of the strong rarefractional phase following the compression shock. This is due to a low cavitation threshold at the metallized PVDF surface.

Further investigations concern a capacitively, via deionized water, coupled PVDF membrane hydrophone ${ }^{15}$ (sensitive diameter $1 \mathrm{~mm}$ ), providing an increased lifetime due to minor cavitation damage. This hydrophone had been compared to the fibre-optic probe hydrophone (Figures 14, 15), positioned successively in the focus of

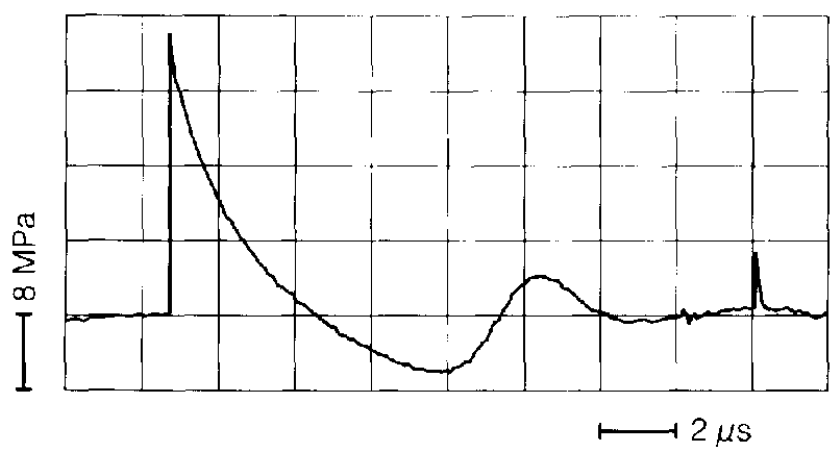

Figure 12 Signal corresponding to Figure 11 , but PVDF membrane hydrophone; high frequency linear extrapolation sets extrema to $+28 /-6.6 \mathrm{MPa} \pm 10 \%$, negative pressure cut off by cavitation

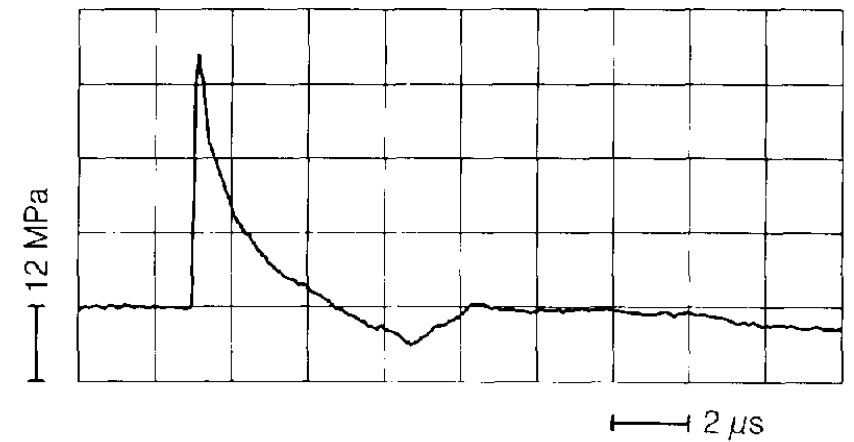

Figure 13 Signal corresponding to Figure 11, but commercial PVDF needle hydrophone ${ }^{6}$; deconvolution sets extrema to $+31 /-6.1 \mathrm{MPa} \pm 15 \%$, negative pressure cut off by cavitation

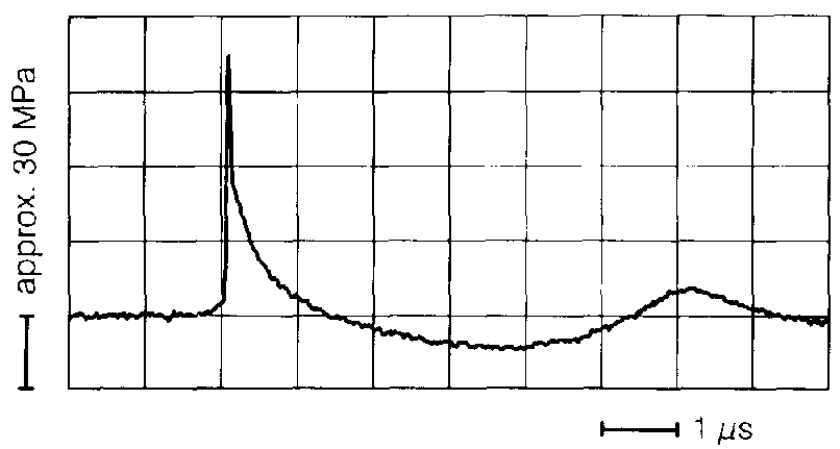

Figure 14 Siemens Lithostar Plus, Overhead Module: focal signal at maximum setting, recorded by a fibre-optic probe hydrophone linearization and high frequency linear extrapolation sets extrema to $+72 /-12 \mathrm{MPa} \pm 5 \%$ 


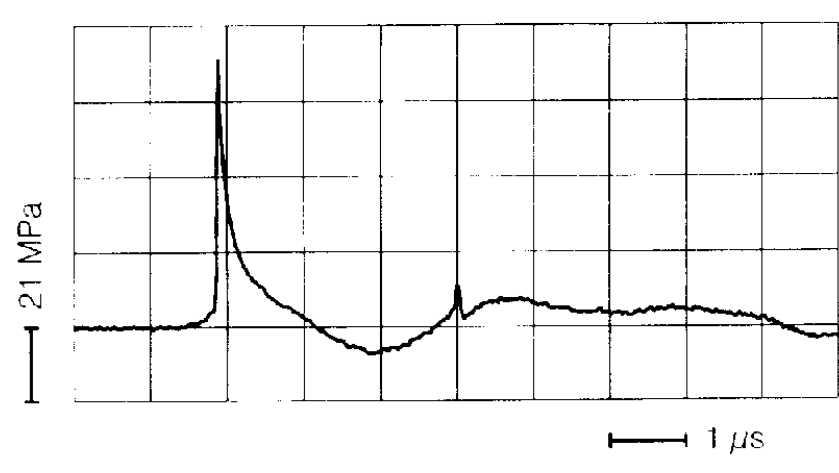

Figure 15 Signal corresponding to Figure 14, but capacitively coupled, via deionized water, PVDF membrane hydrophone ${ }^{15}$; high frequency linear extrapolation sets extrema to $+68 /-7.3 \mathrm{MPa} \pm 10 \%$, negative pressure cut off by cavitation

a commercial lithotripter (Siemens Lithostar Plus. Overhead Module).

Considering positive pressure data, good agreement within calibration accuracy has been found, while significant differences are observed in negative pressure detection. According to Figures 14 and 15 it is obvious. that the non-metallized, capacitively coupled membrane hydrophone cuts off a major part of the negative pressure phase. From literature it is known that the polar character of fused silica gives a strong adhesion to water, even stronger than the cohesion of water itself ${ }^{30}$. Hence, the fibre-optic probe hydrophone provides successful detection of the highest negative pressure amplitudes $(-15 \mathrm{MPa})$ we have observed so far. For clarity it should be pointed out, that cavitation at or close to the fibre endface will cause a strong positive jump in reflectivity. usually saturating the photodetector.

\section{Durability and long term stability}

The static reflection $R_{0}+S$, monitored by the dc-level $l_{i}$ of the photodetector, performs a sensitive detection mechanism for possible fibre damage. Fast and easy repair is possible just by cleaving the libre again. Long-term stability is confirmed by the perpendicular endface itself, implying precise clcaving tools.

To detect high negative pressure waveforms without distortion, the lateral polymer coating of the fibre, due to its low cavitation threshold. has to be removed at a distance of several millimetres from the libre endface. Fibre damage had been demonstrated to arise from cavitation events in this uncoated section ${ }^{14}$. Nevertheless, in low cavitation lithotriopter fields, represented for instance by the Siemens Lithostar Plus Overhead Module, the hydrophone lifetime in the focal region at the maximum setting ranged between 500 and 1000 shocks.

In respect of measurements in strong cavitation fields it is desireable to improve the ruggedness properties. Good experiences had been achieved with thin polyurethane coatings. leaving the fibre endface uncovered. However, the bubble growth involved with a lowered cavitation threshold microbends the fibre and the resulting light intensity fluctuations may distort the negative pressure signal.

\section{Further developments}

Covering the fibre endface with a thin layer of appropriatc elastomeric or other materials. the hydrophone sensitivity can be increased. For optically clear silicone rubber. seven times (refer to Figure 5), for opaque silicones already up to 30 times, the sensitivity with respect to the bare fibre in water has been achieved ${ }^{25}$. Thus, modified fibre-optic probe hydrophones will address the low pressure range. competing with piezoelectric polymer hydrophones.

\section{Conclusions}

The fibre-optic probe hydrophone sets a new standard in high intensity ultrasound and shock wave measurement technology. Using this device, for the first time "true" negative pressure waveforms in extracorporeal shock wave lithotripsy can be determined. In viwo measurements are conceivable. Further investigations correlating physical shock wave parameters to stone fragmentation efficiacy and tissue damage will be the content of a subsequent paper.

\section{Acknowledgements}

This work was supported in part by the 'Bundesministerium für Forschung und Technik', Germany, contract number $01 \mathrm{~K} 1 \mathrm{18706/7}$. We thank the medical engineering group, Siemens AG, Erlangen, Germany, for their assistance with the Lithostar and for providing the capacitively coupled membrane hydrophone. The fibreoptic probe hydrophone had been financed with the help of 'Patentstelle für die Deutsche Forschung der Fraunhofer-Gesellschaft ${ }^{-31}$.

\section{References}

1 DeReggi, A.S., Roth, S.C., Kenney, J.M.. Edelman, S. and I larris, G.R. Piezoelectric polymer probe for ultrasonic applications If loust Six Am (1981) 69853859

2 Shotton, K.C.. Bacon, D.R. and Quilliam, R.M. A PVI) membrane hydrophone for operation in the rande $0.5 \mathrm{MH} 71$, 15 MIIz Ultrasonites (1980) 18 123-126

3 Lewin, P.A. Schafer, $M$. and Gilmore, I.M. Miniature piezoelectric polymor ultrasonic hydrophone prohes bitrusmic:s $11981) 19213216$

4 Bacon, D.R. (hatacleristics of a PVDF membrane hydrophone for use in the range $1100 \mathrm{MH}$ IEEE Trans Sonic's Citrasan (1982) $2918 \quad 25$

5 Preston, R.C., Bacon, D.R., Livett, A.I. and Rajendran, K. PVDI membrane hydrophone performance properties and their relevance to the measurement of the acoustic rutput of medical ultrasonic equipment $J$ Phis E: Si Instrum (1983) 16786 . 796

6 Platte, M. A polyvinylidene fluoride needle hydrophone for ultrasonic applications LItrasonics (1985) $23113 \quad 118$ (commercially available from Imotec GmbH, 5102 Würselen, Germany।

? Harris, G.R. Hydrophone measurements in diagnostic ultrasound ficlds IEEE Trans LFFC (1988) $\mathbf{3 5} 87 \cdots 101$

\& Eisenmenger, W. Experimentelle Bestimmung der Stolifrontdicka atus dem akustischen frequenzspek trum clektromagnetisch crzeugter Stobwellen in Flüssigkeiten bei einem Stoßdruckbereich wn 10 atm bis 100) atm Acustica (1964) $14187 \quad 204$

a) Coleman, A.I., Saunders, I.E., Crum, L.A. and Dyson, M. Acoststic civitation generated by an extracorporeal shockwave lithotripter (lirusenend Med Biol (1987) $1369-76$

16 Coleman, A.J. and Saunders, I.E. A survey of the atcoustle output of commercial extracorporcal shock wave lithotripters $/$ terasinul Mol Biol $(1989) 15213227$

11 Müller, M. Comparison of Dornict lithotripters measurement of shock wave tields and fragmentation effectiveness Biomed $T$ echmih $11990) 35250 \quad 262$

12 Folherth, W., Köhler, G., Rohwedder, A. and Matura, E. Pressure distribution and energy flow in the focal region of iwo different electromagnetic shock wave sources $J$ Sione l)is (1992) 417

13 I.ewin, P.A., Schafer. M. and Gilmore, J.M. PVDF sensers for 
quantitative acoustic shock wave measurements In Ultrasonics International 89 Conference Proceedings (1989) 548-553

14 Staudenraus, J. Erzeugung und Ausbreitung freifeldfokussicrter Hochenergiedruckimpulse in Wasser Fortschrittberichte VDI (1991) Reihe $21 \mathrm{Nr} .89$

15 Granz, B. PVDF hydrophone for the measurement of shock wayes IEEE Trans Electrical Insulation (1989) 34 499-502

16 Staudenraus, J., Köhler, M. and Eisenmenger, W. Charakterisierung verschiedener Hydrophone unter Stoßwellenbedingungen in Wasser. In Fortschritte der Akustik-DAGA'9l (1991) 221-224

17 Staudenraus, J. and Eisenmenger, W. Optisches Sondenhydrophon. In Fortschritto der Akustik-DAGA'88 (1988) 467-470

18 Landau, L.D. and Lifschitz, E.M. Lehrbuch der theoretischen Physik Bd VI Hydrodynamik Akademie-Verlag Berlin (1966)

19 Beyer, R.T. Nonlinear Acoustics US Naval Sea Systems Command, Washington D.C. (1974)

20 Müller, M. Stoßwellentokussierung in Wasser Dissertation RWTH Aachen (1987)

21 Davison, L. and Graham R.A. Shock compression of solids Phys Rep (1979) $55255-379$

22 Yadav, H.S., Murty, D.S., Veerma, S.N., Sinha, K.H.C., Gupta, B.M. and Chand, D. Measurement of refractive index of waler under high dynamic pressures $J$ Appl Phys (1973) 44 $2197-2200$
23 Rosen, J.S. The refractive indices of alcohol, water, and their mixtures at high pressures J Opt Soc Am (1947) 37 932-938

24 Barker, L.M. and Hollenbach, R.E. Shock wave studies of PMMA fused silica, and sapphire $J$ Appl Phys (1970) 41 4208-4226

25 Staudenraus, J. and Eisenmenger, W. Optisches Sondenhydrophon für Ultraschall- und Stoßwellenmessungen mit einem Meßbereich von 0.1 bar bis 1000 bar. In Fortschritte der Akustik-DAGA $y_{2}$ (1992) 301-304

26 Phillips, R.L. Proposed fiber-optic acoustical probe Opt lett (1980) 5 318-320

27 Bucaro, J.A., Lagakos, N., Cole, J.H. and Giallorenzi, T.G. Fiber optic acoustic transduction. In Physical Acoustics Vol XVI (Ed Mason, W.) Academic Press (1982) 385-457

28 Eisenmenger, W. Elektromagnetische Erzeugung von ebenen Druckstößen in Flüssigkeiten Acustica (1962) 12 (Akustische Beihefte Heft 1) 185-202

29 Eisenmenger, W. Eine Kontrollschallquelle für breitbandige Mikrofone zur Messung von Druckimpulsen in Flüssigkeiten Acustica (1962) $12165-172$

30 Strube, H.W. and Lauterborn, W. Untersuchung der Kavitationskeime an der Grenzfläche Quarzglas Wasser nach der Zentrifugenmethode Z. angew. Physik (1970) 29 349-357

31 Eisenmenger, W. and Staudenraus, J. Sampling hydrophone United States Patent (1991) Patent Number 5,010,248 